\title{
Observation of Antiferromagnetic Resonance in an Organic Superconductor
}

Torrance, J. B.; Pedersen, H. J.; Bechgaard, K.

Published in:

Physical Review Letters

Link to article, DOI:

10.1103/PhysRevLett.49.881

Publication date:

1982

Document Version

Publisher's PDF, also known as Version of record

Link back to DTU Orbit

Citation (APA):

Torrance, J. B., Pedersen, H. J., \& Bechgaard, K. (1982). Observation of Antiferromagnetic Resonance in an Organic Superconductor. Physical Review Letters, 49(12), 881-884. https://doi.org/10.1103/PhysRevLett.49.881

\section{General rights}

Copyright and moral rights for the publications made accessible in the public portal are retained by the authors and/or other copyright owners and it is a condition of accessing publications that users recognise and abide by the legal requirements associated with these rights.

- Users may download and print one copy of any publication from the public portal for the purpose of private study or research.

- You may not further distribute the material or use it for any profit-making activity or commercial gain

- You may freely distribute the URL identifying the publication in the public portal

If you believe that this document breaches copyright please contact us providing details, and we will remove access to the work immediately and investigate your claim. 


\title{
Observation of Antiferromagnetic Resonance in an Organic Superconductor
}

\author{
J. B. Torrance ${ }^{(\mathrm{a})}$ and H. J. Pedersen ${ }^{(\mathrm{b})}$ \\ Physics Laboratory III, Technical University of Denmark, DK-2800 Lyngby, Denmark \\ and \\ K. Bechgaard \\ Department of General and Organic Chemistry, H. C. Oersted Institute, DK-2100 Copenhagen, Denmark \\ (Received 27 May 1982)
}

\begin{abstract}
Anomalous microwave absorption has been observed in the organic superconductor $\mathrm{TMTSF}_{2} \mathrm{AsF}_{6}$ (TMTSF: tetramethyltetraselenafulvalene) below its metal-nonmetal transition near $12 \mathrm{~K}$. This absorption is unambiguously identified as antiferromagnetic resonance by the excellent agreement between a spin-wave calculation and the pronounced dependence on temperature and magnetic field orientation. This result dramatically confirms earlier indications of magnetic ordering or spin-density waves and, furthermore, indicates that the spin-density-wave amplitude is considerably larger than previous estimates of $<1 \%$.
\end{abstract}

PACS numbers: $76.50 .+\mathrm{g}, 72.15 . \mathrm{Nj}, 75.50 . \mathrm{Ee}$

The series of conducting organic compounds ${ }^{1}$ $\mathrm{TMTSF}_{2} M \mathrm{~F}_{6}$, with $M=\mathrm{P}, \mathrm{As}, \mathrm{Ta}, \mathrm{Sb}$, and probably $\mathrm{Nb}$, each appear to exhibit the same unusual and unique behavior ${ }^{2-6}$ : at atmospheric pressure, they are metallic with the conductivity along the stack rising from $\sim 10^{3} \Omega^{-1} \mathrm{~cm}^{-1}$ at $300 \mathrm{~K}$ to $\sim 10^{5}$ $\Omega^{-1} \mathrm{~cm}^{-1}$ near $20 \mathrm{~K}$, below which there is a transition to a nonmetallic state. Under pressure, the temperature $(12-18 \mathrm{~K})$ of this transition is rapidly decreased toward $1 \mathrm{~K}$. Above 6.5-11 kbar (depending on the compound), the nonmetal state has disappeared and the material has become superconducting with $T_{c} \sim 1 \mathrm{~K}$. In this paper, we shall focus on the nature of the nonmetallic state at amospheric pressure of $\mathrm{TMTSF}_{2}-$ $\mathrm{AsF}_{6}$, in which we have observed antiferromagnetic resonance (AFMR). This observation represents the culmination of the growing evidence that in the nonmetallic phase these materials have an antiferromagnetic or spin-density-wave (SDW) ground state: Initially, it was found ${ }^{1}$ that the $\mathrm{PF}_{6}$ salt showed no large decrease in the highfield static susceptibility below its metal-nonmetal transition, contrary to what is found for
Peierls transitions. Pedersen, Scott, and Bechgaard, ${ }^{4}$ however, found that the electron-spin resonance $(E P R)$ intensity did vanish in this nonmetallic phase. Susceptibility measurements by Scott, Pedersen, and Bechgaard ${ }^{7}$ and by Mortensen and co-workers ${ }^{8,9}$ revealed the field dependence and anisotropy of $\chi$ as arising from a spinflip transition, often found in classical antiferromagnets. Other evidence has come from measurements of EPR at high-power levels, ${ }^{10}$ and from NMR on both $\mathrm{Se}^{11}$ and protons. ${ }^{11,12}$ The experiments reported here clearly and dramatically confirm these earlier indications. Furthermore, they make it possible to obtain a quantitative estimate of the magnetic anisotropy, which suggests that the amplitude of the SDW is considerably larger than the previous estimate ${ }^{11}$ of $<1 \%$. It is also important to mention parallel measurements by Walsh et al. ${ }^{13}$ which appear to reveal AFMR, and hence SDW, in the related compound $\mathrm{TMTSF}_{2} \mathrm{ClO}_{4}$.

AFMR is well known in insulating systems with localized spins. For a linear chain ${ }^{14}$ of such spins, $S_{i}$, the behavior is well described by using a spin Hamiltonian, such as

$$
\mathscr{H}=+2 J \sum_{i} \overrightarrow{\mathrm{S}}_{i} \cdot \overrightarrow{\mathrm{S}}_{i+1}-g \mu \overrightarrow{\mathrm{H}} \cdot \sum_{i} \overrightarrow{\mathrm{S}}_{i}+E \sum_{i}\left({S_{i}}^{z}{S_{i+1}}^{z}-S_{i}{ }^{y} S_{i+1}{ }^{y}\right)-D \sum_{i} S_{i}{ }^{x} S_{i+1}{ }^{x} \text {, }
$$

where $J$ is the antiferromagnetic interaction between the spins along the chains. Since the structure of these materials is triclinic, we have included the most general form of the anisotropy, using $D$ and $E$ as parameters, where $D$ represents the hard-axis anisotropy and $J, D, E>0$. Note that the easy, intermediate, and hard axes are $z, y$, and $x$, respectively, and the special cases of $D=E$ and $E=0$ correspond to the uni - axial and easy-plane limits, respectively. (We shall find that $\mathrm{TMTSF}_{2} \mathrm{AsF}_{6}$ is near the latter limit.) The Hamiltonian (1) is usually used for localized spins, but in $\mathrm{TMTSF}_{2} \mathrm{AsF}_{6}$ the spins are clearly not localized on individual sites. For example, in the nonmetallic phase at $4 \mathrm{~K}$, the conductivity is still $\sim 10^{2} \Omega^{-1} \mathrm{~cm}^{-1}$. It is impor tant to recognize that Eq. (1) also applies to the 
spins in a partially filled Hubbard model with strong Coulomb interactions ${ }^{15}$ and to other general spin systems. ${ }^{16}$ Thus, we empirically use Eq. (1), with empirical parameters, and make a conventional spin-wave calculation.

The experiment is performed by placing a single crystal (or pair of aligned crystals) of $\mathrm{TMTSF}_{2} \mathrm{AsF}_{6}(\sim 500 \mu \mathrm{g})$ into a $Q$-band microwave cavity and measuring the power absorbed in the sample. An external magnetic field, $H$, is applied and the sample absorbs when the frequency of one of the AFMR modes is raised by $H$ up to the $Q$-band frequency $(35 \mathrm{GHz})$. The cavity absorption is recorded as a function of the external field, as in Fig. 1(b), showing examples of the AFMR absorption, which is weak and broad ( 1 kOe wide). The resonance behavior is then characterized by the dependence on the magnitude and orientation of $H_{\mathrm{r} \text { es }}$ required for resonance. A discussion of the AFMR results is best divided into analyzing separately the results for the three principal field orientations.

For the external field applied along the easy axis ( $z \sim b^{\prime}$ from Ref. 9), the spin-wave predictions are shown in Fig. 1(a). At zero field there are two AFMR modes $\Omega_{+}$and $\Omega_{-}$:

$$
\begin{aligned}
& \hbar^{2} \Omega_{+}{ }^{2}=(D+E) \pi^{2} J, \\
& \hbar^{2} \Omega_{-}{ }^{2}=2 E \pi^{2} J,
\end{aligned}
$$

where we have included two factors of $\pi / 2$ to account for the zero-point spin fluctuations. of $S=\frac{1}{2}$ in one dimension. ${ }^{16}$ (In the special case of uniaxial anisotropy, $D=E$ and these modes would be degenerate at $H=0$.) In the presence of an applied field $H \| z$, these modes shift as shown. The lower resonance is driven to zero frequency at $H=H_{s f}$, the spin-flop field, and the spins flop from being along $z$ to being along $y$. At $T=0$, the theory predicts $\gamma H_{\text {sf }}=\Omega_{-}$, where $\gamma$ is the gyro-
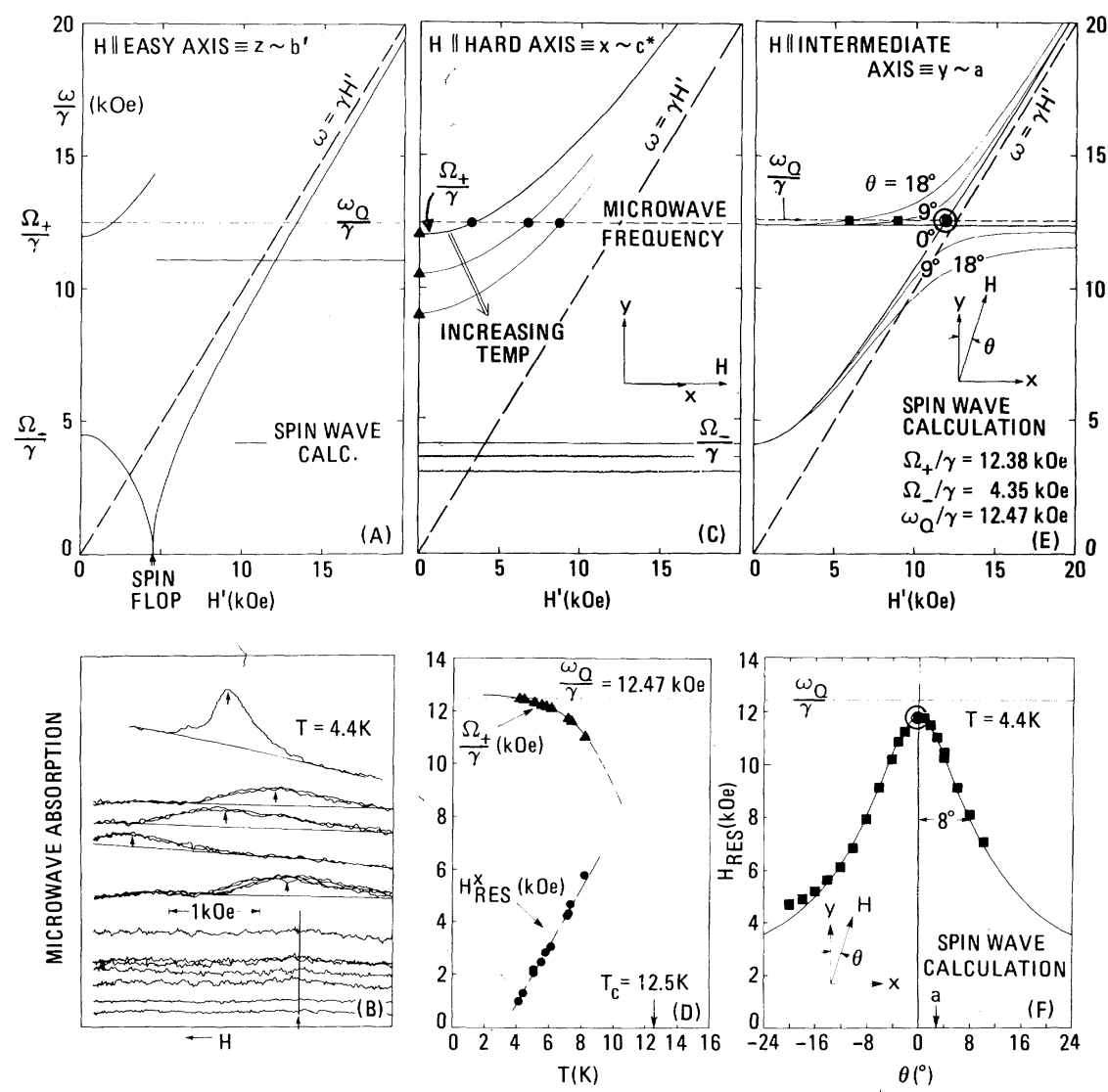

FIG. 1. (a), (c), and (e) Calculated dependence of spin-wave frequencies for $H$ along easy $(z)$, hard $(x)$, and intermediate $(y)$ magnetic anisotropy axes, respectively; (b) typical absorption vs field scan, showing observed resonances; (d) observed resonance field (circles) vs temperature for $H$ along the hard axis, which gives the temperature dependence of $\Omega_{+}$(triangles) via Eq. (3); (f) angular variation of observed (squares) and calculated (solid curve) resonance field for $H$ near intermediate axis $\left(H^{\prime} \equiv g H / 2\right)$. 
magnetic ratio. $H_{s f}$ has been measured ${ }^{9}$ from the field dependence of $\chi$ to be $\sim 4.5 \mathrm{kOe}$. In our measurements for $H \| z$, we have not found the absorption expected [Fig. 1(a)] for $H>H_{s f}$, but we have seen the absorption near $H=0$ associated with the upper mode.

The absorption of this upper mode is seen more clearly for $H$ along the hard direction [Fig. 1(c)], in which case the upper branch increases in frequency, while the lower branch remains field independent. In $\mathrm{TMTSF}_{2} \mathrm{AsF}_{6}$ at $4 \mathrm{~K}, \Omega_{+}$is accidentally slightly above the $Q$-band microwave frequency. In order to decrease $\Omega_{+}$so that the resonance could be observed away from $H \sim 0$, it was necessary to increase the temperature. Thus, the resonance field, $H_{\text {res }}{ }^{x}$, was measured versus $T$ and is shown by the circles in Fig. 1(d). From these data and the resonance condition

$$
\omega_{Q}{ }^{2}=\Omega_{+}{ }^{2}(T)+\left[\gamma^{x} H_{\text {res }}{ }^{x}(T)\right]^{2},
$$

we can calculate $\Omega_{+}(T)$, shown as the triangles, in Fig. 1(d). Extrapolating these values to $T=0$, we obtain an ambiguous measure of $\Omega_{+}(0) / \gamma$ $=12.6 \mathrm{kOe}$. We also learn that the hard axis is close to $c^{*}$, as had been suggested by Mortensen, Tomkiewicz, and Bechgaard. ${ }^{9}$

Finally, the case of the external field applied along the intermediate $(y)$ axis is shown in Fig. 1(e). Here the upper mode is field independent, while the frequency of the lower mode increases with increasing field. In this configuration, a resonance is observed [Fig. $1(\mathrm{f})$ ] at $11.8 \mathrm{kOe}$ at $4.4 \mathrm{~K}$. Using a relation similar to Eq. (3), we find $\Omega_{-} / \gamma=4.4 \mathrm{kOe}$, which compares favorably with the value ${ }^{9}$ of $H_{\mathrm{sf}}=4.5 \pm 0.3 \mathrm{kOe}$.

The angular dependence in the $a-c$ plane [Fig. $1(f)]$ does not have the simple $\cos \theta$ or $1 / \cos \theta$ behavior, but is more pronounced, with a sharp drop in the resonance field to half of its value when only $10^{\circ}$ away from the $y$ axis. Equally striking are the results from the calculation: for $H$ in the $x-y$ plane, the two modes hybridize and become mixed modes as seen in Fig. 1(e). The hybridization gives rise to the calculated angular dependence shown as the solid line in Fig. 1(f). The excellent agreement for such pronounced features as the angular dependence and the temperature dependence [Fig. 1(d)] insure the validity and uniqueness of the identification and interpretation of these modes as AFMR. They also demonstrate the appropriateness of this spinwave theory and the use of Eq. (1) for empirically describing the results. A somewhat similar resonance has been discovered in $\mathrm{TMTSF}_{2} \mathrm{ClO}_{4}$ by Walsh et al., ${ }^{13}$ in a parallel study, although the angular dependence of the $\mathrm{ClO}_{4}$ compound is less pronounced and the analysis is complicated by significant deviations of the principal magnetic axes from the crystallographic axes.

The principal result of the AFMR experiment on $\mathrm{TMTSF}_{2} \mathrm{AsF}_{6}$ is the values measured for the two resonance modes at zero field:

$$
\begin{aligned}
& \Omega_{+}=(12.6 \mathrm{kOe}) / \gamma=35.3 \mathrm{GHz}=1.18 \mathrm{~cm}^{-1} ; \\
& \Omega_{-}=(4.4 \mathrm{kOe}) / \gamma=12.3 \mathrm{GHz}=0.41 \mathrm{~cm}^{-1} .
\end{aligned}
$$

The value of the empirical coefficient of $\vec{S} \cdot \vec{S}$ in Eq. (1), $J$, is related ${ }^{16}$ to the magnitude of the spin susceptibility by the relation $J \chi=2 N_{0} \mu_{B}{ }^{2} / \pi^{2}$, independent of the assumptions of the origin of $\mathcal{H}$, Eq. (1). Using the experimental values for $\Omega_{+}$, $\Omega_{-}$, and $\chi=1.25 \times 10^{-4} \mathrm{emu} / \mathrm{mole}$ at $20 \mathrm{~K},{ }^{9}$ we obtain from Eq. (2)

$$
\begin{aligned}
& J_{\text {expt }}=604 \mathrm{~K}, \quad D_{\text {expt }}=4.5 \times 10^{-4} \mathrm{~K}, \\
& E_{\text {expt }}=0.3 \times 10^{-4} \mathrm{~K}
\end{aligned}
$$

as the experimental values for the empirical parameters in Eq. (1).

Since Fig. 1 shows such a clear observation of AFMR with such quantitative agreement between Eq. (1) and experiment, one might hope to be able to draw some quantitative conclusions about such controversial questions as the amplitude of the SDW and the relative magnitude of the Coulomb interaction $(U)$ and the bandwidth $(4 t)$ in $\mathrm{TMTSF}_{2} \mathrm{AsF}_{6}$. Unfortunately, this is not at all easy since the results in Fig. 1 are general consequences ${ }^{16}$ of the long-wavelength, quasihydrodynamic solutions to Eq. (1) and are not dependent on assumptions of the amplitude of SDW or of the detailed spin structure. That is, they would have been seen if the SDW amplitude were $10^{-6}$ or 1 . The information about the electronic interactions does not come from the existence of the AFMR itself, but from the magnitude of the parameters. The magnitude of $\chi$, however, is not very sensitive $^{17}$ (in this case) to the value of $U / 4 t$, since it can be accounted for by a range of possible values of $U(0<U<1 \mathrm{eV})$ with a reasonable estimate for $4 t \sim 1 \mathrm{eV}$.

The values of $D$ and $E$ are much more sensitive to these interactions. If we assume that this anisotropy is primarily due to dipole-dipole interactions between spins, we can compare calculated $D$ and $E$ with the experimental values. For the maximum amplitude SDW, with spins localized on every other TMTSF molecule, Metzger ${ }^{18}$ has calculated the contribution to the di- 
polar anisotropy from a pair of localized spins along the stack. He finds a hard axis along $a$ and $D_{\text {calc }}=59 \times 10^{-4} \mathrm{~K}$ with a much smaller $E$ term. Since the magnitude of $D$ should go as the square of the SDW amplitude, a SDW with $\sim 1 \%$ amplitude, as previously estimated, ${ }^{11}$ would be expected to give $D \sim 0.06 \times 10^{-4} \mathrm{~K}$ or about 700 times smaller than $D_{\text {expt }}$. Thus, it appears that a much larger amplitude $(10 \%-20 \%)$ SDW is necessary in order to account for the order of magnitude of the large anisotropies determined from the AFMR measurements.

In conclusion, we have observed unusual microwave absorption in the nonmetallic phase of $\mathrm{TMTSF}_{2} \mathrm{AsF}_{6}$. The strong temperature and angular dependences, fitted by a conventional spinwave theory, clearly prove that this absorption is antiferromagnetic resonance, observed here for the first time in an organic solid. This observation provides clear confirmation of earlier suggestions and indications that the nonmetallic phase in the TMTSF $X_{2} \mathrm{~F}_{6}$ salts is antiferromagnetic. The existence of this magnetic ground state emphasizes the importance of Coulomb interactions in these as well as other organic solids. ${ }^{19}$ In this case, what is particularly remarkable is that this antiferromagnetic phase exists adjacent to a superconducting phase. This fact emphasizes the unique nature of the properties of these materials, and raises questions about the mechanism of the observed superconductivity.

We gratefully acknowledge helpful and enjoyable discussions with M. C. Cross, T. Guldbransen, C. S. Jacobsen, R. M. Metzger, K. Mortensen, J. C. Scott, and W. M. Walsh.

\footnotetext{
(a)Permanent address: IBM Research Laboratory, San Jose, Cal. 95193.
}

(b) Present address: Danfoss A/s, Building E14/S12, DK-6430 Nordborg, Denmark.

${ }^{1} \mathrm{TMTSF}=$ tetramethyltetraselenafulvalene.

${ }^{2}$ K. Bechgaard, C. S. Jacobsen, K. Mortensen, H. J. Pedersen, and N. Thorup, Solid State Commun. 33, 1119 (1980).

${ }^{3}$ D. Jerome, A. Mazaud, M. Rebault, and Ko Bechgaard, J. Phys. (Paris), Lett. 41, L95 (1980).

${ }^{4}$ H. J. Pedersen, J. C. Scott, and K. Bechgaard, Solid State Commun. 35, 207 (1980).

${ }^{5}$ S. S. P. Parkin, F. Cruezet, M. Ribault, D. Jerome, K. Bechgaard, and J. M. Fabre, Mol. Cryst. Liq. Cryst. 79, 249 (1982).

${ }^{6}$ K. Bechgaard, Mol. Cryst. Liq. Cryst. 79, 1 (1982).

7J. C. Scott, H. J. Pedersen, and K. Bechgaard, Phys. Rev. Lett. 45, 2125 (1980).

${ }^{8}$ K. Mortensen, Y. Tomkiewicz, T. D. Schultz, and E. M. Engler, Phys. Rev. Lett. 46, 1234 (1981).

${ }^{9} \mathrm{~K}$. Mortensen, Y. Tomkiewic z, and K. Bechgaard, Phys. Rev. B 25, 3319 (1982).

${ }^{10}$ W. M.Walsh, F. Wudl, G. A. Thomas, D. Nalewajek, J. J. Hauser, P. A. Lee, and T. O. Poehler, Phys. Rev. Lett. 45,829 (1980).

${ }^{11}$ A. Andrieux, D. Jerome, and K. Bechgaard, J. Phys. (Paris), Lett. 42, L87 (1981).

${ }^{12}$ J. C. Scott, H. J. Pedersen, and K. Bechgaard, Phys. Rev. B 24, 475 (1981).

${ }^{13}$ W. M. Walsh, Jr., F. Wudl, E. Aharon-Shalom, L. W. Rupp, Jr., J. M. Vanderberg, K. Andres, and J. B. Torrance, following Letter [Phys. Rev. Lett. $\underline{49}$, 885 (1982)].

${ }^{14}$ Note that we have left out the very weak interchain exchange interactions which stabilize the ordering of the antiferromagnetic state.

${ }^{15}$ D。 J. Klein and W. A. Seitz, Phys. Rev. B 10,3217 (1974).

${ }^{16}$ M. C. Cross, in Proceedings of the Sixteenth International Conference on Low Temperature Physics, LOS Angeles, 1981 (North-Holland, Amsterdam, to be published).

${ }^{17} \mathrm{~J}$. B. Torrance, Y. Tomkiewicz, and B. D. Silverman, Phys. Rev. B 15, 4738 (1977).

${ }^{18}$ R. M. Metzger, J. Chem. Phys. 75, 482 (1981).

${ }^{19} \mathrm{~J}$. B. Torrance, in Chemistry and Physics of OneDimensional Metals : NATO Advanced Study Institute Series, Vol. 25, edited by H. J. Keller (Plenum, New York, 1977), p. 137. 